Vietnam Journal of Mechanics, VAST, Vol.39, No. 4 (2017), pp. $327-340$

DOI:10.15625/0866-7136/9641

\title{
A CLOSED-FORM SOLUTION FOR FREE VIBRATION OF MULTIPLE CRACKED TIMOSHENKO BEAM AND APPLICATION
}

\author{
Nguyen Tien Khiem ${ }^{1, *}$, Duong The Hung ${ }^{2}$ \\ ${ }^{1}$ Institute of Mechanics, VAST, 18 Hoang Quoc Viet, Hanoi, Vietnam \\ ${ }^{2}$ Thai Nguyen University of Technology, Vietnam \\ *E-mail: ntkhiem@imech.vast.vn \\ Received April 13, 2017
}

\begin{abstract}
A closed-form solution for free vibration is constructed and used for obtaining explicit frequency equation and mode shapes of Timoshenko beams with arbitrary number of cracks. The cracks are represented by the rotational springs of stiffness calculated from the crack depth. Using the obtained frequency equation, the sensitivity of natural frequencies to crack of the beams is examined in comparison with the Euler-Bernoulli beams. Numerical results demonstrate that the Timoshenko beam theory is efficiently applicable not only for short or fat beams but also for the long or slender ones. Nevertheless, both the theories are equivalent in sensitivity analysis of fundamental frequency to cracks and they get to be different for higher frequencies.
\end{abstract}

Keywords: Timoshenko beams, multiple cracked beams, natural frequencies, sensitivity analysis.

\section{INTRODUCTION}

About a century has passed from the date when Timoshenko Beam Theory (TBT) was proposed and although it is not straightforward as the classical Euler-Bernoulli Beam Theory (EBT) the TBT is not less popular nowadays than the classical one [1]. Generalized by taking into account the shear deformation and rotary inertia the TBT has extended applicability of beam theory to analysis of short or fat beams which are more widely encountered in the practice of structural engineering. Basics for dynamic analysis of Timoshenko beams are provided in numerous publications on structural dynamics, for example, the References [2-4]. Recently, because of potential hazards produced by presence of a crack in a structure, dynamic analysis of cracked structures gets an enormous attention of researchers and engineers. Numerous methods were proposed for modal analysis and crack detection based on the classical EBT [5-10]. Among the obtained

(C) 2017 Vietnam Academy of Science and Technology 
results it is worth to note that a closed-form solution for the vibration mode of EulerBernoulli beams with multiple cracks was conducted and used for obtaining an explicit expression for characteristic equation of the beam [11,12]. Vibration of cracked Timoshenko beams has been studied by numerous authors in [13-18] among that the studies by Li [15] and Aydin [18] are noteworthy by their achievements in modal analysis of cracked Timoshenko beams. Some particular solutions of the crack detection problem for Timoshenko beam were obtained earlier in $[13,14,17]$ and recently by Khaji and his coworkers in $[19,20]$ using the conventional methods.

In the present paper a closed-form solution for free vibration of Timoshenko beam with arbitrary number of cracks is conducted and used for constructing an explicit expression for both frequency equation and mode shape of the beam. Using the obtained frequency equation, the sensitivity of natural frequencies to crack of the beams is examined in comparison with the Euler-Bernoulli beams [12]. Numerical results demonstrate that the Timoshenko beam theory is efficiently applicable not only for short or thick beams but also for the long or slender one. Nevertheless, both the theories are equivalent in sensitivity analysis of fundamental frequency to cracks and they get to be different in the analysis of higher frequencies.

\section{A CLOSED-FORM SOLUTION FOR FREE VIBRATION OF TIMOSHENKO BEAM WITH MULTIPLE CRACKS}

Consider a uniform beam of length $\ell$; material density $(\rho)$; elasticity $(E)$ and shear (G) modulus; area $A=b \times h$ and moment of inertia $I=b h^{3} / 12$ of cross section. Assuming first order shear deformation (Timoshenko) theory of beam, the displacement field in cross-section at $x$ and height $z$ from the neutral axis is

$$
u(x, z, t)=u_{0}(x, t)-z \theta(x, t) ; w(x, z, t)=w_{0}(x, t),
$$

with $u_{0}(x, t), w_{0}(x, t), \theta(x, t)$ being respectively the displacements and slope at central axis.

Using the constituting equations

$$
\varepsilon_{x}=\partial u_{0} / \partial x-z \partial \theta / \partial x ; \gamma_{x z}=\partial w_{0} / \partial x-\theta ; \quad \sigma_{x}=E \varepsilon_{x} ; \tau_{x z}=\kappa G \gamma_{x z}
$$

and Hamilton principle, the equations for free vibration of the beam can be established as

$$
\rho A \ddot{w}-\kappa G A\left(w^{\prime \prime}-\theta^{\prime}\right)=0 ; \rho I \ddot{\theta}-E I \theta^{\prime \prime}-\kappa G A\left(w^{\prime}-\theta\right)=0,
$$

where $\ddot{w}=\partial^{2} w / \partial t^{2}, w^{\prime}=\partial w / \partial x, w^{\prime \prime}=\partial^{2} w / \partial x^{2}$ and $\ddot{\theta}=\partial^{2} \theta / \partial t^{2}, \theta^{\prime}=\partial \theta / \partial x, \theta^{\prime \prime}=$ $\partial^{2} \theta / \partial x^{2}$.

Seeking solution of (3) in the form

$$
w(x, t)=W(x) e^{i \omega t} ; \theta(x, t)=\Theta(x) e^{i \omega t},
$$

one gets

$$
\omega^{2} \rho W(x)+\kappa G\left(W^{\prime \prime}-\Theta^{\prime}\right)=0 ; \quad \omega^{2} \rho I \Theta(x)+E I \Theta^{\prime \prime}(x)+\kappa G A\left(W^{\prime}-\Theta\right)=0 .
$$

Furthermore, it is assumed that the beam has been cracked at positions $e_{j}, j=$ $1, \ldots, n$ and the cracks are modeled by rotational springs of stiffness $K_{j}$ calculated from 
crack depth [8]. Therefore, conditions that must be satisfied at the crack section are

$$
\begin{aligned}
& W\left(e_{j}+0\right)=W\left(e_{j}-0\right) ; \Theta\left(e_{j}+0\right)=\Theta\left(e_{j}-0\right)+M\left(e_{j}\right) / K_{j} ; \\
& Q\left(e_{j}+0\right)=Q\left(e_{j}-0\right)=Q\left(e_{j}\right) ; M\left(e_{j}+0\right)=M\left(e_{j}-0\right)=M\left(e_{j}\right),
\end{aligned}
$$

where $N, Q, M$ are respectively internal axial, shear forces and bending moment at section $x$

$$
M=E I \Theta_{x}^{\prime} ; Q=\kappa G A\left(W_{x}^{\prime}-\Theta\right) .
$$

Substituting (7) into (6) one can rewrite the latter conditions as

$$
\begin{aligned}
& W\left(e_{j}+0\right)=W\left(e_{j}-0\right)=W\left(e_{j}\right) ; \Theta_{x}^{\prime}\left(e_{j}+0\right)=\Theta_{x}^{\prime}\left(e_{j}-0\right)=\Theta^{\prime}\left(e_{j}\right) ; \\
& \Theta\left(e_{j}+0\right)=\Theta\left(e_{j}-0\right)+\gamma_{j} \Theta_{x}^{\prime}\left(e_{j}\right) ; W_{x}^{\prime}\left(e_{j}+0\right)=W_{x}^{\prime}\left(e_{j}-0\right)+\gamma_{j} \Theta_{x}^{\prime}\left(e_{j}\right) ; \gamma_{j}=E I / K_{j} .
\end{aligned}
$$

Seeking solution of Eq. (5) in the form $W_{0}(x)=C_{w} e^{\lambda x}, \Theta_{0}(x)=C_{\theta} e^{\lambda x}$ one is able to obtain so-called characteristic equation

$$
\begin{gathered}
\lambda^{4}+b \lambda^{2}-c=0, \\
b=\alpha(1+\beta) ; c=\alpha(\tau-\alpha \beta) ; \alpha=\rho \omega^{2} / E ; \quad \beta=E / \kappa G ; \tau=A / I .
\end{gathered}
$$

This is a square algebraic equation with respect to $\eta=\lambda^{2}$ that can be elementarily solved to give roots

$$
\eta_{1}=\left(-b+\sqrt{b^{2}+4 c}\right) / 2 ; \eta_{2}=-\left(b+\sqrt{b^{2}+4 c}\right) / 2 .
$$

Note that in the case if $c=0$ the Eq. (9) has the roots

$$
\lambda_{1,2}= \pm i \sqrt{b}= \pm i \omega \sqrt{\rho(1+\beta) / E} ; \lambda_{3,4}=0 .
$$

This occurs when $\omega=\omega_{c}=\sqrt{12 \kappa G / \rho h^{2}}$ acknowledged as cut-off frequency of the beam. Otherwise, the Eq. (9) has the roots

$$
\lambda_{1,2}= \pm k_{1} ; \quad \lambda_{3,4}= \pm i k_{2} ; \quad k_{1}=\sqrt{\left(\sqrt{b^{2}+4 c}-b\right) / 2}, \quad k_{2}=\sqrt{\left(\sqrt{b^{2}+4 c}+b\right) / 2},
$$

for frequency less than cut-off one, $\omega<\omega_{c}=\sqrt{\kappa G A / \rho I}$. Since the cut-off frequency is very high, vibration of the beam is often investigated in the lower frequency range $\left(0, \omega_{c}\right)$. Thus, in the frequency range, general continuous solution of Eq. (5) can be represented as

$$
\begin{gathered}
W_{0}(x)=C_{1} \cosh k_{1} x+C_{2} \sinh k_{1} x+C_{3} \cos k_{2} x+C_{4} \sin k_{2} x, \\
\Theta_{0}(x)=r_{1} C_{1} \sinh k_{1} x+r_{1} C_{2} \cosh k_{1} x+r_{2} C_{3} \sin k_{2} x-r_{2} C_{4} \cos k_{2} x, \\
r_{1}=\left(\rho \omega^{2} / \kappa G k_{1}+k_{1}\right) ; r_{2}=\left(\rho \omega^{2} / \kappa G k_{2}-k_{2}\right) .
\end{gathered}
$$

Particularly, solution (14) and (15) satisfying the conditions

$$
W_{0}(0)=0 ; W_{0}^{\prime}(0)=1 ; \Theta_{0}(0)=1 ; \Theta_{0}^{\prime}(0)=0,
$$

is

$$
\begin{gathered}
S_{w}(x)=S_{1} \sinh k_{1} x+S_{2} \sin k_{2} x ; S_{\theta}(x)=r_{1} S_{1} \cosh k_{1} x-r_{2} S_{2} \cos k_{2} x, \\
S_{1}=\left(r_{2}+k_{2}\right) /\left(r_{1} k_{2}+r_{2} k_{1}\right) ; S_{2}=\left(r_{1}-k_{1}\right) /\left(r_{1} k_{2}+r_{2} k_{1}\right) .
\end{gathered}
$$


Using obtained above particular solution, general solution of Eq. (5) satisfying conditions (8) at cracks is represented by

$$
\begin{gathered}
W(x, \omega)=C_{1} W_{1}\left(k_{1}, x\right)+C_{2} W_{2}\left(k_{1}, x\right)+C_{3} W_{3}\left(k_{2}, x\right)+C_{4} W_{4}\left(k_{2}, x\right), \\
\Theta(x, \omega)=C_{1} \Theta_{1}\left(k_{1}, x\right)+C_{2} \Theta_{2}\left(k_{1}, x\right)+C_{3} \Theta_{3}\left(k_{2}, x\right)+C_{4} \Theta_{4}\left(k_{2}, x\right),
\end{gathered}
$$

where

$$
\begin{aligned}
& W_{1}(x)=\cosh k_{1} x+\sum_{j=1}^{n} \mu_{1 j} K_{w}\left(x-e_{j}\right) ; \quad W_{2}(x)=\sinh k_{1} x+\sum_{j=1}^{n} \mu_{2 j} K_{w}\left(x-e_{j}\right), \\
& W_{3}(x)=\cos k_{2} x+\sum_{j=1}^{n} \mu_{3 j} K_{w}\left(x-e_{j}\right) ; \quad W_{4}(x)=\sin k_{2} x+\sum_{j=1}^{n} \mu_{4 j} K_{w}\left(x-e_{j}\right), \\
& \Theta_{1}(x)=r_{1} \sinh k_{1} x+\sum_{j=1}^{n} \mu_{1 j} K_{\theta}\left(x-e_{j}\right) ; \Theta_{2}(x)=r_{1} \cosh k_{1} x+\sum_{j=1}^{n} \mu_{2 j} K_{\theta}\left(x-e_{j}\right), \\
& \Theta_{3}(x)=r_{2} \sin k_{2} x+\sum_{j=1}^{n} \mu_{3 j} K_{\theta}\left(x-e_{j}\right) ; \Theta_{4}(x)=-r_{2} \cos k_{2} x+\sum_{j=1}^{n} \mu_{4 j} K_{\theta}\left(x-e_{j}\right) \text {, } \\
& K_{w}(x)=\left\{\begin{array}{ll}
0: & x<0 ; \\
S_{w}(x): & x \geq 0 ;
\end{array} \quad K_{w}^{\prime}(x)= \begin{cases}0: & x<0 \\
S_{w}^{\prime}(x): & x \geq 0\end{cases} \right. \\
& K_{\theta}(x)=\left\{\begin{array}{ll}
0: & x<0 ; \\
S_{\theta}(x): & x \geq 0 ;
\end{array} \quad K_{\theta}^{\prime}(x)= \begin{cases}0: & x<0 \\
S_{\theta}^{\prime}(x): & x \geq 0\end{cases} \right. \\
& \mu_{k j}=\gamma_{j}\left\{L_{k}\left(e_{j}\right)+\sum_{i=1}^{j-1} \mu_{k i} S_{\theta}^{\prime}\left(e_{j}-e_{i}\right)\right\} ; k=1,2,3,4 ; j=1,2, \ldots, n . \\
& L_{1}(x)=k_{1} r_{1} \cosh k_{1} x ; L_{2}(x)=k_{1} r_{1} \sinh k_{1} x ; L_{3}(x)=k_{2} r_{2} \cos k_{2} x ; L_{4}(x)=k_{2} r_{2} \sin k_{2} x \text {. }
\end{aligned}
$$

\section{NATURAL FREQUENCIES AND MODE SHAPES}

In this section, frequency equation is obtained for beam with classical boundary conditions such as simply supported (SS), clamped (CC) beam and cantilever (clamped and free $(\mathrm{CF})$ end beam). The boundary conditions are expressed as follow:

- For SS-beam: $W(0)=M(0)=W(\ell)=M(\ell)=0$. In this case

$$
\begin{aligned}
& C_{1} W_{1}\left(k_{1}, 0\right)+C_{2} W_{2}\left(k_{1}, 0\right)+C_{3} W_{3}\left(k_{2}, 0\right)+C_{4} W_{4}\left(k_{2}, 0\right)=0 \Rightarrow C_{1}+C_{3}=0, \\
& C_{1} \Theta_{1}^{\prime}\left(k_{1}, 0\right)+C_{2} \Theta_{2}^{\prime}\left(k_{1}, 0\right)+C_{3} \Theta_{3}^{\prime}\left(k_{2}, 0\right)+C_{4} \Theta_{4}^{\prime}\left(k_{2}, 0\right)=0 \Rightarrow r_{1} k_{1} C_{1}+r_{2} k_{2} C_{3}=0,
\end{aligned}
$$

from that we have got $C_{1}=C_{3}=0$ and

$$
C_{2} W_{2}\left(k_{1}, \ell\right)+C_{4} W_{4}\left(k_{2}, \ell\right)=0 ; \quad C_{2} \Theta_{2}^{\prime}\left(k_{1}, \ell\right)+C_{4} \Theta_{4}^{\prime}\left(k_{2}, \ell\right)=0 .
$$

Therefore, frequency equation is obtained in the form $W_{2}\left(k_{1}, \ell\right) \Theta_{4}^{\prime}\left(k_{2}, \ell\right)$ $-W_{4}\left(k_{2}, \ell\right) \Theta_{2}^{\prime}\left(k_{1}, \ell\right)=0$ or

$$
d_{S S}(\omega)+\sum_{j=1}^{n}\left[\mu_{2 j} \bar{S}_{2}\left(\ell-e_{j}\right)+\mu_{4 j} \bar{S}_{4}\left(\ell-e_{j}\right)\right]+\sum_{j, k=1}^{n} \mu_{2 j} \mu_{4 j} \bar{S}_{24}\left(e_{j}, e_{k}\right)=0,
$$


where

$$
\begin{aligned}
& d_{S S}(\omega)=\left(r_{2} k_{2}-r_{1} k_{1}\right) \sinh k_{1} \ell \sin k_{2} \ell, \\
& \bar{S}_{2}\left(\ell-e_{j}\right)=\left[r_{2} k_{2} S_{w}\left(\ell-e_{j}\right)-S_{\theta}^{\prime}\left(\ell-e_{j}\right)\right] \sin k_{2} \ell, \\
& \bar{S}_{4}\left(\ell-e_{j}\right)=\left[S_{\theta}^{\prime}\left(\ell-e_{j}\right)-r_{1} k_{1} S_{w}\left(\ell-e_{j}\right)\right] \sinh k_{1} \ell, \\
& \bar{S}_{24}\left(e_{j}, e_{k}\right)=S_{w}\left(\ell-e_{j}\right) S_{\theta}^{\prime}\left(\ell-e_{k}\right)-S_{w}\left(\ell-e_{k}\right) S_{\theta}^{\prime}\left(\ell-e_{j}\right) .
\end{aligned}
$$

For uncracked beam, the frequency equation (24) is reduced to $\sin k_{2} \ell=0$ that leads to $k_{2} \ell=j \pi ; j=1,2,3, \ldots$ and in the case of single crack, $n=1$, Eq. (25) is

$$
f_{S S}(\omega)+\gamma g_{s S}(e, \omega)=0,
$$

with

$$
f_{S S}(\omega)=\sinh k_{1} \ell \sin k_{2} \ell,
$$

$g_{s s}(e, \omega)=r_{2} k_{2} S_{1} \sinh k_{1} e \sinh k_{1}(\ell-e) \sin k_{2} \ell+r_{1} k_{1} S_{2} \sin k_{2} e \sin k_{2}(\ell-e) \sinh k_{1} \ell$.

The latter equation has been obtained in [19].

- For CC-beam: $W(0)=\Theta(0)=W(\ell)=\Theta(\ell)=0$. The conditions at $x=0$ lead to $C_{1}+C_{3}=0$ and $r_{1} C_{2}-r_{2} C_{4}=0$. So that

$$
W(x)=C_{1} L_{w 1}(x)+C_{2} L_{w 2}(x) ; \Theta(x)=C_{1} L_{\theta 1}(x)+C_{2} L_{\theta 2}(x)
$$

with

$$
\begin{array}{r}
L_{w 1}(x)=L_{01}(x)+\sum_{j=1}^{n} \bar{\mu}_{1 j} K_{w}\left(x-e_{j}\right) ; \quad L_{w 2}(x)=L_{02}(x)+\sum_{j=1}^{n} \bar{\mu}_{3 j} K_{w}\left(x-e_{j}\right) ; \\
L_{\theta 1}(x)=L_{03}(x)+\sum_{j=1}^{n} \bar{\mu}_{1 j} K_{\theta}\left(x-e_{j}\right) ; \quad L_{\theta 2}(x)=L_{04}(x)+\sum_{j=1}^{n} \bar{\mu}_{3 j} K_{\theta}\left(x-e_{j}\right) ;
\end{array}
$$

$L_{01}=\cosh k_{1} x-\cos k_{2} x ; L_{02}=r_{2} \sinh k_{1} x+r_{1} \sin k_{2} x ; \bar{\mu}_{1 j}=\mu_{1 j}-\mu_{3 j} ; \bar{\mu}_{3 j}=r_{2} \mu_{1 j}+r_{1} \mu_{3 j} ;$

$$
L_{03}=r_{1} \sinh k_{1} x-r_{2} \sin k_{2} x ; L_{04}=r_{1} r_{2}\left(\cosh k_{1} x-\cos k_{2} x\right) .
$$

Therefore, frequency equation for clamped beam is derived from the conditions

$$
W(\ell)=C_{1} L_{w 1}(\ell)+C_{2} L_{w 2}(\ell)=0 ; \quad \Theta(\ell)=C_{1} L_{\theta 1}(\ell)+C_{2} L_{\theta 2}(\ell)=0
$$

as $L_{w 1}(\ell) L_{\theta 2}(\ell)-L_{w 2}(\ell) L_{\theta 1}(\ell)=0$ or

$$
d_{C C}(\omega)+\sum_{j=1}^{n}\left[\bar{\mu}_{1 j} \bar{S}_{1}\left(\ell-e_{j}\right)+\bar{\mu}_{3 j} \bar{S}_{3}\left(\ell-e_{j}\right)\right]+\sum_{j, k=1}^{n} \bar{\mu}_{1 j} \bar{\mu}_{3 k} \bar{S}_{13}\left(e_{j}, e_{k}\right)=0,
$$

where

$$
\begin{aligned}
& d_{C C}(\omega)=L_{01}(\ell) L_{04}(\ell)-L_{02}(\ell) L_{03}(\ell), \\
& \bar{S}_{1}\left(\ell-e_{j}\right)=L_{04}(\ell) S_{w}\left(\ell-e_{j}\right)-L_{02}(\ell) S_{\theta}\left(\ell-e_{j}\right), \\
& \bar{S}_{3}\left(L-e_{j}\right)=L_{01}(\ell) S_{\theta}\left(\ell-e_{j}\right)-L_{03}(\ell) S_{w}\left(\ell-e_{j}\right), \\
& \bar{S}_{13}\left(e_{j}, e_{k}\right)=S_{w}\left(\ell-e_{j}\right) S_{\theta}\left(\ell-e_{k}\right)-S_{w}\left(\ell-e_{k}\right) S_{\theta}\left(\ell-e_{j}\right) .
\end{aligned}
$$

In the case of single crack, Eq. (31) is simplified [19]

$$
d_{C C}(\omega)+\gamma\left\{\left[L_{1}(e)-L_{3}(e)\right] \bar{S}_{1}(\ell-e)+\left[r_{2} L_{1}(e)+r_{1} L_{3}(e)\right] \bar{S}_{3}(\ell-e)\right\}=0,
$$


or

$$
f_{c c}(\omega)+\gamma g_{c c}(e, \omega)=0
$$

with

$$
\begin{aligned}
f_{c c}(\omega) & =2 r_{1} r_{2}\left(1-\cosh k_{1} \ell \cos k_{2} \ell\right)+\left(r_{2}^{2}-r_{1}^{2}\right) \sinh k_{1} \ell \sin k_{2} \ell ; \\
g_{c c}(e, \omega)= & {\left[L_{1}(e)-L_{3}(e)\right]\left\{L_{04}(\ell) S_{w}(\ell-e)-L_{02}(\ell) S_{\theta}(\ell-e)\right\}+} \\
& +\left[r_{2} L_{1}(e)+r_{1} L_{3}(e)\right]\left\{L_{01}(\ell) S_{\theta}(\ell-e) s-L_{03}(\ell) S_{w}(\ell-e)\right\} .
\end{aligned}
$$

- For CF-beam: $W(0)=\Theta(0)=M(\ell)=Q(\ell)=0$. It is found above that conditions for clamp at $x=0$ lead the solutions (19), (20) to expressions (27), so that conditions for free end at $x=\ell$ now yield

$$
C_{1}\left[L_{w 1}^{\prime}(\ell)-L_{\theta 1}(\ell)\right]+C_{2}\left[L_{w 2}(\ell)-L_{\theta 2}(\ell)\right]=0 ; \quad C_{1} L_{\theta 1}^{\prime}(\ell)+C_{2} L_{\theta 2}^{\prime}(\ell)=0,
$$

that allow one to obtain frequency equation for CF-beam in the form

$$
\left[L_{w 1}^{\prime}(\ell) L_{\theta 2}^{\prime}(\ell)-L_{w 2}^{\prime}(\ell) L_{\theta 1}^{\prime}(\ell)\right]-\left[L_{\theta 1}(\ell) L_{\theta 2}^{\prime}(\ell)-L_{\theta 2}(\ell) L_{\theta 1}^{\prime}(\ell)\right]=0 .
$$

The latter equation can be rewritten as

$$
d_{C F}(\omega)+\sum_{j=1}^{n}\left[\bar{\mu}_{1 j} \bar{G}_{1}\left(\ell-e_{j}\right)+\bar{\mu}_{3 j} \bar{G}_{3}\left(\ell-e_{j}\right)\right]+\sum_{j, k=1}^{n} \bar{\mu}_{1 j} \bar{\mu}_{3 k} \bar{G}_{13}\left(e_{j}, e_{k}\right)=0,
$$

where

$$
\begin{aligned}
& d_{C F}(\omega)=\left[L_{01}^{\prime}(\ell)-L_{03}(\ell)\right] L_{04}^{\prime}(\ell)-\left[L_{02}^{\prime}(\ell)-L_{04}(\ell)\right] L_{03}^{\prime}(\ell), \\
& \bar{G}_{1}\left(\ell-e_{j}\right)=L_{04}^{\prime}(\ell)\left[S_{w}^{\prime}\left(\ell-e_{j}\right)-S_{\theta}\left(\ell-e_{j}\right)\right]-\left[L_{02}^{\prime}(\ell)-L_{04}(\ell)\right] S_{\theta}^{\prime}\left(\ell-e_{j}\right), \\
& \bar{G}_{3}\left(L-e_{j}\right)=\left[L_{01}^{\prime}(\ell)-L_{03}(\ell)\right] S_{\theta}^{\prime}\left(\ell-e_{j}\right)-L_{03}^{\prime}(\ell)\left[S_{w}^{\prime}\left(\ell-e_{j}\right)-S_{\theta}\left(\ell-e_{j}\right)\right], \\
& \left.\bar{G}_{13}\left(e_{j}, e_{k}\right)=\left[S_{w}^{\prime}\left(\ell-e_{j}\right)-S_{\theta}\left(\ell-e_{j}\right)\right] S_{\theta}^{\prime}\left(\ell-e_{k}\right)-\left[S_{w}^{\prime}\left(\ell-e_{k}\right)-S_{\theta}\left(\ell-e_{k}\right)\right]\right] S_{\theta}^{\prime}\left(\ell-e_{j}\right) .
\end{aligned}
$$

Similarly, one can obtain frequency for beam with single crack in the form [19]

$$
d_{C F}(\omega)+\gamma\left\{\left[L_{1}(e)-L_{3}(e)\right] \bar{G}_{1}(\ell-e)+\left[r_{2} L_{1}(e)+r_{1} L_{3}(e)\right] \bar{G}_{3}(\ell-e)\right\}=0,
$$

Solving Eqs. (25), (31) and (36) with respect to $\omega$ gives rise natural frequencies $\omega_{j}, j=1,2,3, \ldots$ Every natural frequency $\omega_{j}$ allows one to calculate first the wave numbers $k_{1 j}, k_{2 j}$ by using (13) and then associated mode shape as

$$
\varphi_{j}(x)=D_{j}\left[W_{4}\left(k_{2 j}, L\right) W_{2}\left(k_{1 j}, x\right)-W_{2}\left(k_{1 j}, L\right) W_{4}\left(k_{2 j}, x\right)\right] .
$$

In latter equation arbitrary constant $D_{j}$ is determined by a chosen condition for normalization.

\section{NUMERICAL ANALYSIS}

To validate the theoretical development, natural frequencies computed by different methods (analytical method [3]; Galerkin's method [17] and the present method) for simply supported beam are compared and given in Tabs. 1-2. The Tables show that the analytical method, Galerkin's and present methods give the same results in computing natural frequencies of intact (uncracked) beam structures with different slenderness ratios. However, disagreement of the methods is apparent when they are applied for 
Table 1. Comparison of frequency parameter $\left(\lambda_{k}=\left[\omega_{k}^{2} \rho A / E I\right]^{1 / 4}\right)$ computed by using different beam theories and methods for simply supported uniform intact beam

\begin{tabular}{|c|c|c|c|c|c|}
\hline Eigenvalue No & 1 & 2 & 3 & 4 & 5 \\
\hline EBT [3] & $\pi$ & $2 \pi$ & $3 \pi$ & $4 \pi$ & $5 \pi$ \\
\hline EBT - Present & 3.1416 & 6.2832 & 9.4248 & 12.5664 & 15.7080 \\
\hline TBT [3] & 3.1155 & 6.0867 & 8.8180 & 11.2766 & 13.4740 \\
\hline TBT - Present & 3.1157 & 6.0907 & 8.8405 & 11.3431 & 13.6132 \\
\hline \multicolumn{6}{|c|}{ Beam parameters: $E=200 \mathrm{GPa} ; \rho=7855 \mathrm{~kg} / \mathrm{m}^{3} ; v=0.3 ;$} \\
$\kappa=5 / 6 ; L=1.0 ; b=0.1 ; h=0.1(\mathrm{~m})$ \\
\hline
\end{tabular}

Table 2. Comparison of natural frequencies computed by using different beam theories and methods for simply supported cracked beam with various slenderness $(L / h)$ and single crack at the beam middle

\begin{tabular}{|c|c|c|c|c|c|c|c|c|}
\hline \multirow{2}{*}{ Frequency No } & \multicolumn{2}{|c|}{1} & \multicolumn{2}{|c|}{2} & \multicolumn{2}{|c|}{3} & \multicolumn{2}{|c|}{4} \\
\hline & $\omega_{0}$ & $\omega_{c} / \omega_{0}$ & $\omega_{0}$ & $\omega_{c} / \omega_{0}$ & $\omega_{0}$ & $\omega_{c} / \omega_{0}$ & $\omega_{0}$ & $\omega_{c} / \omega_{0}$ \\
\hline \multicolumn{9}{|c|}{$L / h=15$} \\
\hline EBT - GM [17] & 303.64 & 0.8836 & 1214.56 & 0.9801 & 2732.77 & 0.9185 & 4808.26 & 0.9673 \\
\hline EBT - Present & 3.64 & 8383 & 1213.10 & 1.0000 & 2732.80 & 0.8740 & 1851.50 & 1.0000 \\
\hline TBT - & 301.34 & 0.8844 & 1179.28 & 0.9806 & 2565.03 & 0.9234 & 4366.67 & 0.9707 \\
\hline TBT - Present & 301.30 & 0.8397 & 1179.30 & 1.0000 & 2565.00 & 0.8827 & 4367.70 & 1.0000 \\
\hline \multicolumn{9}{|c|}{$L / h=10$} \\
\hline EBT - GM [17] & 455.46 & 0.8268 & 1821.85 & 0.9588 & 4099.15 & 0.8906 & 7287.39 & 0.9397 \\
\hline EBT - Present & 455.46 & 0.7319 & 1819.70 & 1.0000 & 4099.20 & 0.8430 & 7277.30 & 1.0000 \\
\hline TBT - GM [17] & 447.84 & 0.8293 & 1710.02 & 0.9613 & 3599.00 & 0.9030 & 5918.77 & 0.9509 \\
\hline TBT - Present & 447.80 & 0.7857 & 1710.00 & 1.0000 & 3599.00 & 0.8628 & 5918.80 & 1.0000 \\
\hline \multicolumn{9}{|c|}{$L / h=5$} \\
\hline EBT - GM [17] & 910.92 & 0.6855 & 3643.72 & 0.8721 & 8198.31 & 0.8245 & 14574.77 & 0.8545 \\
\hline EBT - Present & 910.92 & 0.6631 & 3639.30 & 1.0000 & 8198.30 & 0.7922 & 14555.00 & 1.0000 \\
\hline TBT & 5.01 & 0.6985 & 2959.38 & 0.8936 & 5643.70 & 0.8686 & 8551.50 & 0.9069 \\
\hline TBT - Present & 855.00 & 0.6799 & 2959.40 & 1.0000 & 5643.70 & 0.8484 & 8511.50 & 1.0000 \\
\hline Beam parameters & \multicolumn{8}{|c|}{$\begin{array}{c}E=62.1 \mathrm{GPa} ; G=23.3 \mathrm{Gpa} ; \rho=2770 \mathrm{~kg} / \mathrm{m}^{3} ; v=0.3 \\
\kappa=5 / 6 ; e / L=0.5 ; a / h=0.5\end{array}$} \\
\hline \multicolumn{9}{|c|}{$\begin{array}{c}\text { EBT-Euler Beam Theory; TBT-Timoshenko Beam Theory; GM-Galerkin Method; } \\
\omega_{0} \text { - natural frequency (Rad/s) of intact beam; } \\
\omega_{c} / \omega_{0}-\text { frequency of cracked beam/frequency of intact beam }\end{array}$} \\
\hline
\end{tabular}

cracked beam and miscalculation of Galerkin's method can be observed from that it results in reduction of second and fourth frequencies as the crack appeared at the middle of beam whereas the frequencies should be unchanged due to crack. Finally, it can be seen from Tab. 2 that Timoshenko beam model is more useful to apply for calculating natural frequencies of cracked beam. Note, all the results related to Euler-Bernoulli beam provided herein as EBT-present are obtained for corresponding beam parameters by using the theory developed in Ref. [12].

Effect of slenderness ratio on natural frequencies computed by different beam theories is demonstrated in Tab. 3. The data depicted in Tab. 3 show that Timoshenko beam 
Table 3. Comparison of frequency parameter computed by using different beam theories for simply supported uniform intact beam with various slenderness $(L / h)$

\begin{tabular}{|c|c|c|c|c|c|}
\hline Eigenvalue No & 1 & 2 & 3 & 4 & 5 \\
\hline \multicolumn{7}{|c|}{$L / h=100(L=10, h=0.1)$} \\
\hline Euler-Bernoulli beam [12] & 0.3142 & 0.6283 & 0.9425 & 1.2566 & 1.5708 \\
\hline Timoshenko beam - present & 0.3141 & 0.6281 & 0.9418 & 1.2549 & 1.5675 \\
\hline \multicolumn{7}{|c|}{$L / h=50(L=5, h=0.1)$} \\
\hline Euler-Bernoulli beam [12] & 0.6283 & 1.2566 & 1.8850 & 2.5133 & 3.1416 \\
\hline Timoshenko beam - present & 0.6281 & 1.2549 & 1.8793 & 2.4999 & 3.1157 \\
\hline \multicolumn{7}{|c|}{$L / h=30(L=3, h=0.1)$} \\
\hline Euler-Bernoulli beam [12] & 1.0472 & 2.0944 & 3.1416 & 4.1888 & 5.2360 \\
\hline Timoshenko beam - present & 1.0462 & 2.0866 & 3.1157 & 4.1286 & 5.1213 \\
\hline \multicolumn{7}{|c|}{$L / h=20(L=2, h=0.1)$} \\
\hline Euler-Bernoulli beam [12] & 1.5708 & 3.1416 & 4.7124 & 6.2832 & 7.8540 \\
\hline Timoshenko beam - present & 1.5675 & 3.1157 & 4.6277 & 6.0907 & 7.4963 \\
\hline \multicolumn{7}{|c|}{$L / h=15(L=1.5, h=0.1)$} \\
\hline Euler-Bernoulli beam [12] & 2.0944 & 4.1888 & 6.2832 & 8.3776 & 10.4720 \\
\hline Timoshenko beam - present & 2.0866 & 4.1286 & 6.0907 & 7.9513 & 9.7019 \\
\hline \multicolumn{7}{|c|}{$L / h=10(L=1, h=0.1)$} \\
\hline Euler-Bernoulli beam [12] & 3.1416 & 6.2832 & 9.4248 & 12.5664 & 15.7080 \\
\hline Timoshenko beam - present & 3.1157 & 6.0907 & 8.8405 & 11.3431 & 13.6132 \\
\hline \multicolumn{7}{|c|}{$L / h=5(L=0.5, h=0.1)$} \\
\hline Euler-Bernoulli beam [12] & 6.2832 & 12.5664 & 18.8496 & 25.1328 & 31.4160 \\
\hline Timoshenko beam - present & 6.0907 & 11.3431 & 15.6790 & 19.3142 & 22.4441 \\
\hline Beam parameters: $E=200 \mathrm{GPa} \rho=7855 \mathrm{~kg} / \mathrm{m}^{3} ; v=0.3 ; \kappa=5 / 6 ;$ \\
\hline \multicolumn{7}{|c|}{$\lambda_{k}=\left[\omega_{k}^{2} \rho A / E I\right]^{1 / 4}$} \\
\hline
\end{tabular}

theory gives rise almost the same natural frequencies as the Euler-Bernoulli beam theory for the beams with slenderness ratio greater 20 (acknowledged as long or slender beams). This fact allows one to make a conclusion that Timoshenko beam theory is useful not only for short or thick beams but also for long or slender ones while the Euler-Bernoulli theory is applicable only for the long or slender beams. In case of cracked beam, natural frequencies of Timoshenko beam with single crack computed by the present method are compared to those given in Ref. [19] that are obtained by the conventional transfer matrix method (see Tab. 4). The comparison demonstrates very good agreement of the results, especially, some frequencies are computed identically (when they are unaffected by presence of crack). Thus the proposed in this study method is validated not only in the case of uncracked beam but also for beam with cracks. Furthermore, natural frequencies of cracked Timoshenko beam normalized by those of intact one are computed as function of crack position along the beam span for various slenderness ratios $(10,20,30)$. The frequency ratios (cracked to intact) acknowledged as sensitivity of natural frequencies to 
Table 4. Comparison of natural frequencies with those given in Khaji et al. [19] for Timoshenko beam in case of single crack and various boundary conditions (SS, CC and CF-beams)

\begin{tabular}{|c|c|c|c|c|c|c|c|c|c|c|c|c|}
\hline \multirow{2}{*}{$\begin{array}{c}\text { Freq. } \\
\text { No }\end{array}$} & \multicolumn{3}{|c|}{$L / h=3$} & \multicolumn{3}{|c|}{$L / h=5$} & \multicolumn{3}{|c|}{$L / h=7$} & \multicolumn{3}{|c|}{$L / h=9$} \\
\hline & Present & Khaji & $\%$ & Present & Khaji & $\%$ & Present & Khaji & $\%$ & Present & Khaji & $\%$ \\
\hline \multicolumn{13}{|c|}{ Simply supported beam (SS) } \\
\hline 1 & 6909.92 & 6781.8 & $\underline{1.8}$ & 2919.21 & 2877.3 & $\underline{1.4}$ & 1599.09 & 1580.3 & $\underline{1.1}$ & 1006.68 & 996.9 & $\underline{0.9}$ \\
\hline 2 & 27316.17 & 27316.2 & 0.0 & 12222.26 & 12222.2 & 0.0 & 6803.30 & 6803.3 & 0.0 & 4293.28 & 4293.3 & 0.0 \\
\hline 3 & 42979.87 & 42718.8 & 0.6 & 21132.25 & 21006.4 & 0.6 & 12504.58 & 12430.6 & 0.6 & 8633.80 & 8177.0 & 0.5 \\
\hline 4 & 64534.74 & 64534.6 & 0.0 & 35435.66 & 35435.8 & 0.0 & 21681.69 & 21681.7 & 0.0 & 14556.97 & 14557.0 & 0.0 \\
\hline \multicolumn{13}{|c|}{ Clamped end beam (CC) } \\
\hline 1 & 13700.28 & 13628.4 & 0.5 & 6244.02 & 6205.3 & 0.6 & 3530.10 & 3509.1 & 0.6 & 2255.5 & 2243.2 & 0.5 \\
\hline 2 & 31009.05 & 31009.0 & 0.0 & 15650.89 & 15650.9 & 0.0 & 9335.63 & 9335.6 & 0.0 & 6134.70 & 6134.7 & 0.0 \\
\hline 3 & 44150.76 & 43315.8 & 1.8 & 23731.61 & 23599.0 & 0.5 & 14875.08 & 14796.1 & 0.5 & 10149.80 & 10098.1 & 0.5 \\
\hline 4 & 64534.74 & 64534.6 & 0.0 & 37268.75 & 37268.75 & 0.0 & 23764.42 & 23764.4 & 0.0 & 16475.53 & 16475.5 & 0.0 \\
\hline \multicolumn{13}{|c|}{ Cantilever beam (CF) } \\
\hline 1 & 3157.3 & 3134.9 & 0.7 & 1228.7 & 1222.9 & 0.5 & 645.2 & 642.9 & 0.3 & 396.0 & 394.9 & 0.3 \\
\hline 2 & 12826.6 & 12664.4 & 1.2 & 5954.8 & 5888.2 & 1.1 & 3386.0 & 3352.9 & 0.9 & 2170.8 & 2152.2 & 0.8 \\
\hline 3 & 33422.2 & 33381.4 & 0.1 & 16510.6 & 16504.4 & 0.0 & 9684.8 & 9683.4 & 0.0 & 6296.1 & 6295.6 & 0.0 \\
\hline 4 & 49075.2 & 48898.9 & 0.3 & 5443.6 & 25326.0 & 0.4 & 15583.1 & 15506.8 & 0.5 & 10490.0 & 0438.8 & 0.5 \\
\hline
\end{tabular}

crack are compared to those obtained by using Euler-Bernoulli beam theory and shown in Figs. 1-3 corresponding to the simply supported, clamped-clamped and clampedfree boundary conditions. Obviously, the frequency sensitivities computed for both the beam theories are identical as the slenderness ratio equals to 30 and they get to be apparently deviated for the ratio $L / h=10$. In the latter case, natural frequencies computed by Euler-Bernoulli beam theory are more sensitive to crack. Note, that the sensitivity of fundamental frequency of cantilever beam is independent on which beam theory is applied.

To investigate combined effect of the beam theories, slenderness ratio and multiple cracks on natural frequencies, the frequency parameter $\lambda_{k}=\left[\omega_{k}^{2} \rho A / E I\right]^{1 / 4}, k=$ $1,2,3,4,5$ are computed for various slenderness $L / h=5,10,20(h=0.2,0.1,0.05, L=1)$, number of cracks $n=0,1,2,3$ using the different beam theories (EBT and TBT). Deviation of the frequency parameters computed by the EBT and TBT is calculated and measured in percent $(\%)$.

Obviously, the frequency parameter computed for Euler-Bernoulli intact beam is independent on the beam thickness $(h)$ for the beam length $(L)$ fixed, as can be seen in Tab. 5, it is dependent only on the beam length. However, as a crack occurred in beam the parameter decreases with increasing beam thickness and number of cracks. Unlikely, the frequency parameter of Timoshenko beam is always decreasing as the beam thickness and number of cracks are growing. Difference between the beam theories measured by 

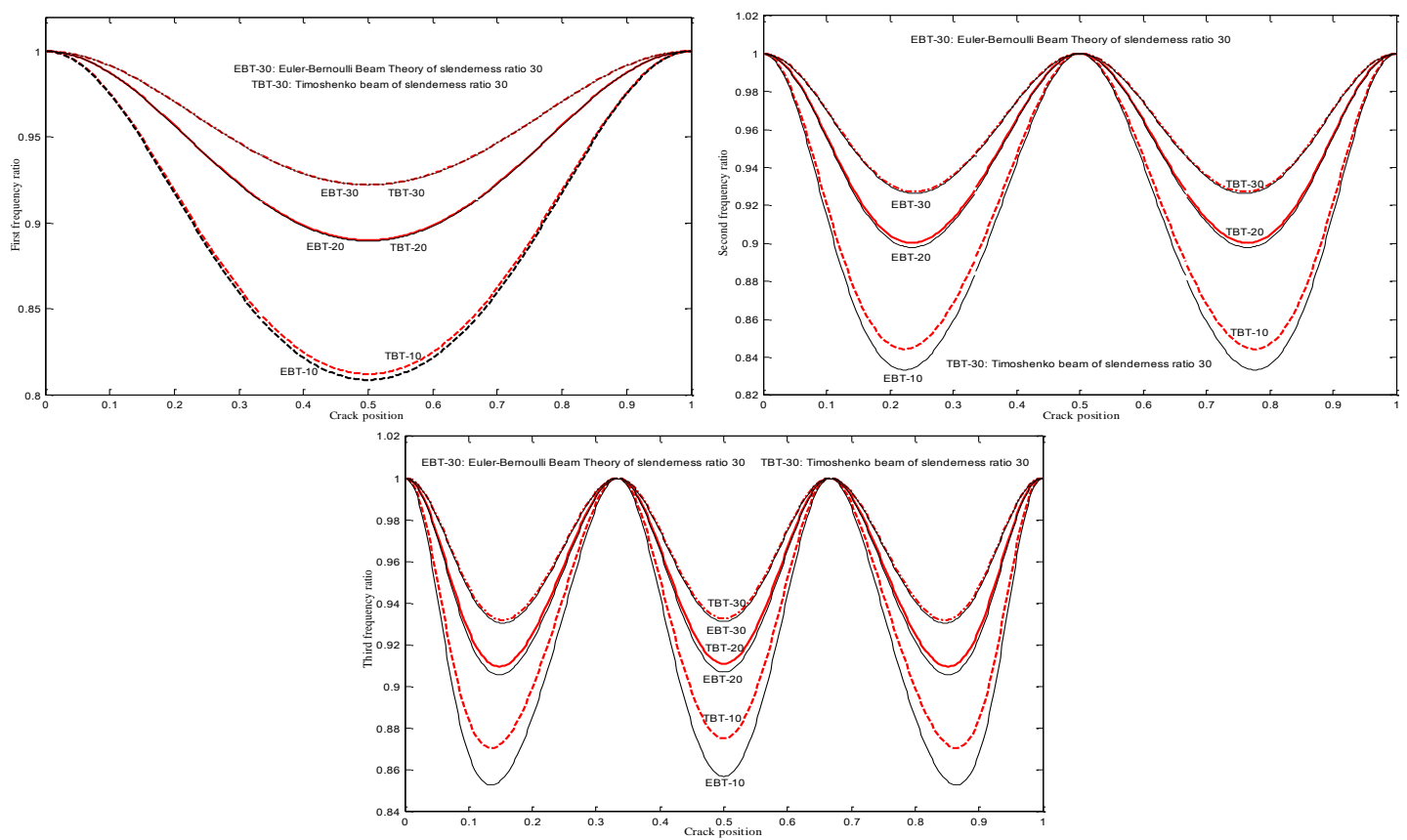

Fig. 1. Change (induced by single crack of depth $30 \%$ ) in natural frequencies computed by EBT and TBT in various slenderness of uniform simply supported beam
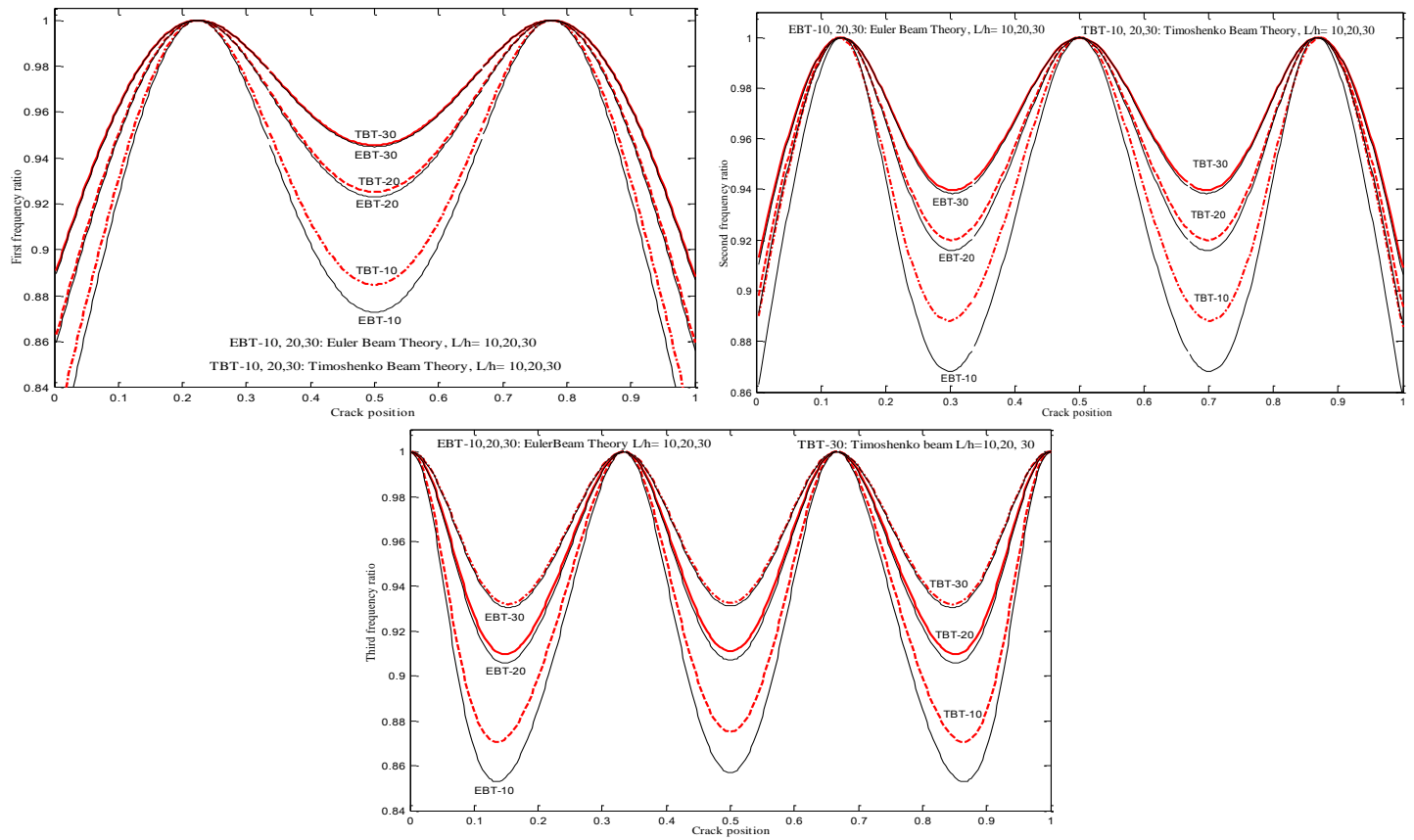

Fig. 2. Change (induced by single crack of depth $30 \%$ ) in natural frequencies computed by EBT and TBT in various slenderness of uniform clamped end beam 

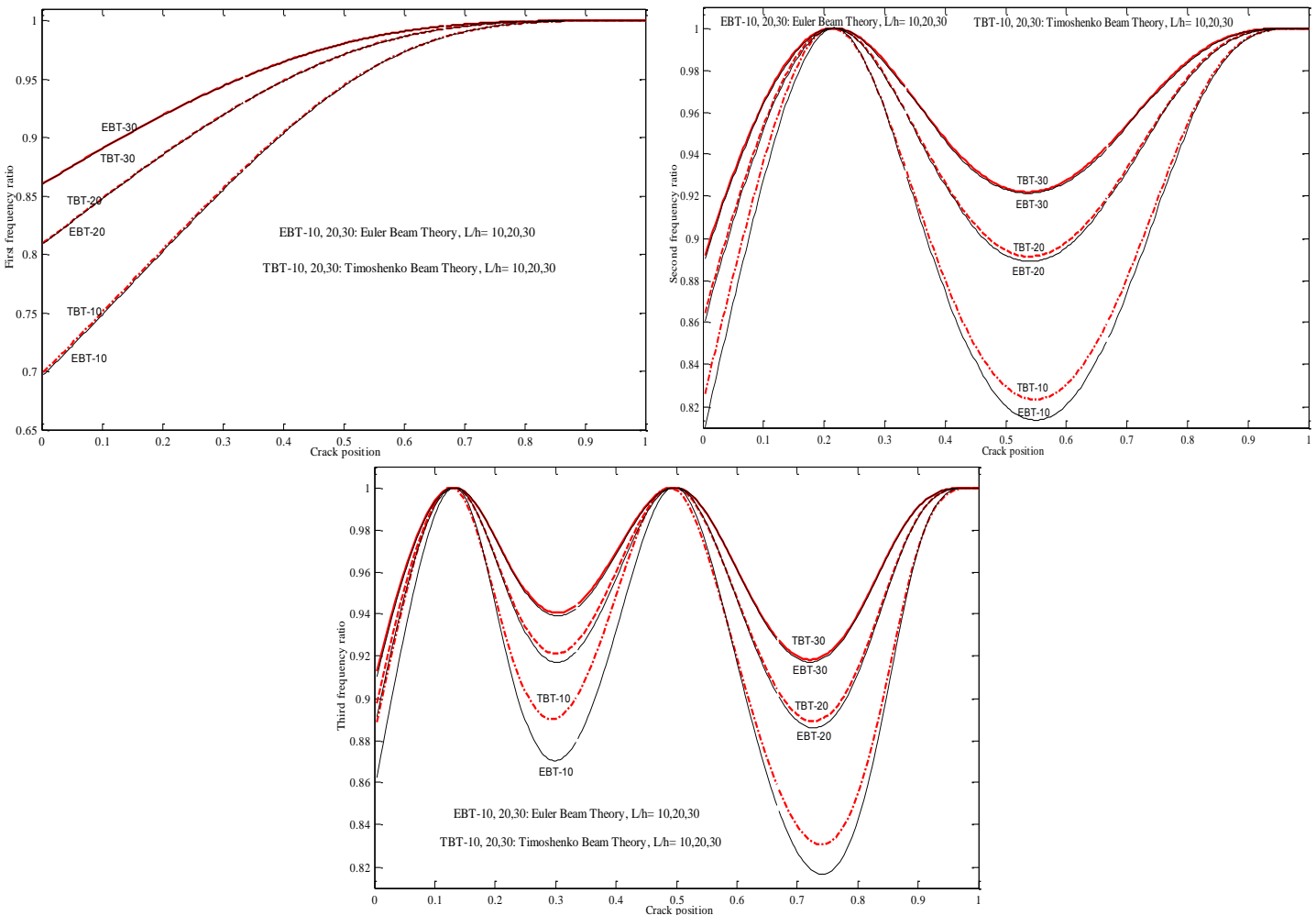

Fig. 3. Change (induced by single crack of depth $30 \%$ ) in natural frequencies computed by EBT and TBT in various slenderness of uniform cantilevered beam

deviation of the frequency parameter is significant and rising with mode number. However, the deviation decreases not only as usually for increasing slenderness ratio but also when number of cracks rises, except the case of fundamental frequency.

Table 5. Comparison of natural frequencies computed by Timoshenko and Euler-Bernoulli beams with various number of cracks (equal depth $30 \%$ ), slenderness ratio and boundary conditions

\begin{tabular}{|c|c|c|c|c|c|c|c|c|c|c|c|c|c|}
\hline \multirow{2}{*}{$L / h$} & \multirow{2}{*}{$\begin{array}{l}\text { No. } \\
\text { Freq. }\end{array}$} & \multicolumn{3}{|c|}{ No crack } & \multicolumn{3}{|c|}{ Single crack at $(1 / 6)$} & \multicolumn{3}{|c|}{ Double crack at $(1 / 6 ; 1 / 2)$} & \multicolumn{3}{|c|}{ Triple crack at $(1 / 6 ; 1 / 2 ; 5 / 6)$} \\
\hline & & TBT & EBT & $\delta(\%)$ & TBT & EBT & $\delta(\%)$ & TBT & EBT & $\delta(\%)$ & TBT & EBT & $\delta(\%)$ \\
\hline \multicolumn{14}{|c|}{ Simply supported beam } \\
\hline \multirow{5}{*}{5} & 1 & .0453 & 3.1416 & 3.0 & 2.8769 & 2.9545 & 2.6 & 2.7202 & 2.5438 & -6.9 & 2.6269 & 2.4783 & -6.0 \\
\hline & 2 & 5.6716 & 6.2832 & 9.7 & 5.0824 & 5.4966 & 7.5 & 5.0342 & .3615 & 6.1 & 4.6248 & 4.9248 & 6.1 \\
\hline & 3 & 7.8395 & 9.4248 & 16.8 & 7.2807 & .5199 & 4.5 & .9992 & .7166 & 9.3 & 6.2958 & 6.5851 & 4.4 \\
\hline & 4 & 9.6571 & 12.5664 & 23.1 & 9.3386 & 11.9524 & 21.8 & 9.3165 & 11.8327 & 21.2 & 8.9259 & 11.1797 & 20.1 \\
\hline & 5 & 1.2220 & 15.7080 & 28.5 & 11.1138 & 15.4740 & 28.1 & 10.8266 & 4.2329 & 23.9 & 10.6653 & 13.7340 & 22.3 \\
\hline
\end{tabular}




\begin{tabular}{|c|c|c|c|c|c|c|c|c|c|c|c|c|c|}
\hline \multirow{5}{*}{10} & 1 & 3.1157 & 3.1416 & 0.8 & 3.0202 & 3.0431 & 0.7 & 2.9163 & 2.7670 & -5.4 & 2.8493 & 2.7157 & -4.9 \\
\hline & 2 & . 0907 & .2832 & .0 & .6488 & .7939 & 2.5 & 5.6352 & 5.7569 & 2.1 & 5.2980 & 5.4130 & 2.1 \\
\hline & 3 & 8.8405 & 9.4248 & 6.2 & 8.2847 & 8.7481 & 5.3 & 8.0456 & 8.1319 & 1.1 & 7.4634 & 7.4260 & -0.5 \\
\hline & 4 & 11.3431 & 12.5664 & 9.7 & 10.9777 & 12.0688 & 9.0 & 10.9611 & 12.0125 & 8.7 & 10.5372 & 11.4781 & 8.2 \\
\hline & 5 & 13.6132 & 15.7080 & 13.3 & 13.4888 & 15.5165 & 13.0 & 13.1670 & 14.5410 & 9.4 & 12.9827 & 14.1942 & 8.5 \\
\hline \multirow{5}{*}{20} & 1 & 3.1350 & 3.1416 & 0.2 & 3.0849 & 3.0911 & 0.2 & 3.0250 & 2.9253 & -3.4 & 2.9839 & 2.8906 & -3.2 \\
\hline & 2 & 6.2314 & 6.2832 & 0.8 & 5.9637 & 6.0074 & 0.7 & 5.9611 & 6.0000 & 0.6 & 5.7353 & 5.7730 & 0.6 \\
\hline & 3 & 9.2554 & .4248 & 1.8 & 8.8385 & 8.9811 & 1.5 & 8.6795 & 8.5669 & -1.3 & 8.2761 & 8.1421 & -1.6 \\
\hline & 4 & 12.1813 & 12.5664 & 3.0 & 11.8588 & 12.2080 & 2.8 & 11.8522 & 12.1895 & 2.7 & 11.4967 & 11.8076 & 2.6 \\
\hline & 5 & 14.9926 & 15.7080 & .5 & 14.8731 & 15.5678 & 4.4 & 14.6178 & 14.8937 & 1.8 & 14.4615 & 4.6810 & 1.5 \\
\hline \multicolumn{14}{|c|}{ Clamped beam } \\
\hline \multirow{5}{*}{5} & 1 & 4.2420 & 4.7300 & 10.3 & 4.2028 & 4.6668 & 9.9 & 4.0313 & 4.1188 & 2.1 & 3.9593 & 3.9522 & -0.1 \\
\hline & 2 & 6.4179 & 7.8532 & 18.2 & 6.3354 & 7.7581 & 18.3 & 6.3327 & 7.7544 & 18.3 & 6.2671 & 7.6823 & 18.4 \\
\hline & 3 & 8.2853 & 10.9956 & 24.6 & 7.9838 & 10.2966 & 22.4 & 7.6980 & 9.4961 & 18.9 & 7.4429 & 9.1124 & 18.3 \\
\hline & 4 & 9.9037 & 14.1372 & 29.9 & 9.6529 & 13.2401 & 27.1 & 9.6393 & 13.0124 & 25.9 & 9.3531 & 12.3131 & 24.0 \\
\hline & 5 & 11.3487 & 17.2788 & 34.3 & 11.2675 & 16.6622 & 32.3 & 11.0219 & 15.5326 & 29.0 & 10.9035 & 14.4893 & 24.7 \\
\hline \multirow{5}{*}{10} & 1 & 4.5795 & 4.7300 & 3.2 & 4.5418 & 4.6862 & 3.1 & 4.4159 & 4.3432 & -1.6 & 4.3631 & 4.2598 & -2.4 \\
\hline & 2 & 7.3312 & 7.8532 & 6.6 & 7.2712 & 7.7887 & 6.6 & 7.2707 & 7.7875 & 6.6 & 7.2196 & 7.7334 & 6.6 \\
\hline & 3 & 9.8561 & 10.9956 & 10.3 & 9.5050 & 10.4967 & 9.4 & 9.2695 & 9.8594 & 5.9 & 9.0049 & 9.5540 & 5.7 \\
\hline & 4 & 12.1454 & 14.1372 & 14.1 & 11.7489 & 13.4203 & 12.4 & 11.7274 & 13.3097 & 11.8 & 11.3212 & 2.7100 & 10.9 \\
\hline & 5 & 14.2324 & 17.2788 & 17.6 & 14.0334 & 16.7587 & 16.2 & 13.7334 & 15.8277 & 13.2 & 13.4489 & 15.0624 & 10.7 \\
\hline \multirow{5}{*}{20} & 1 & 4.6899 & 4.7300 & 0.8 & 4.6639 & 4.7029 & 0.8 & 4.5917 & 4.5064 & -1.9 & 4.5605 & 4.4663 & -2.1 \\
\hline & 2 & 7.7035 & 7.8532 & 1.9 & 7.6652 & 7.8140 & 1.9 & 7.6651 & 7.8137 & 1.9 & 7.6303 & 7.7783 & 1.9 \\
\hline & 3 & 10.6401 & 10.9956 & 3.2 & 10.3589 & 10.6819 & 3.0 & 10.1896 & 10.2332 & 0.4 & 9.9690 & 10.0127 & 0.4 \\
\hline & 4 & 13.4611 & 14.1372 & 4.7 & 3.0478 & 13.6321 & 4.3 & 13.0363 & 13.5950 & 4.1 & 12.6374 & 13.1440 & 3.8 \\
\hline & 5 & 16.1590 & 17.2788 & 6.5 & 15.8783 & 16.8852 & 5.9 & 15.6257 & 16.2090 & 3.6 & 15.2800 & 15.7069 & 2.7 \\
\hline \multicolumn{14}{|c|}{ Cantilever beam } \\
\hline \multirow{5}{*}{5} & 1 & 1.8466 & 1.8751 & 1.5 & 1.5168 & 1.5303 & 0.9 & 1.5038 & 1.4918 & -0.8 & 1.5031 & 1.4912 & -0.8 \\
\hline & 2 & 4.2853 & 4.6941 & 8.7 & 4.2661 & 4.6437 & 8.1 & 3.9871 & 3.8567 & -3.4 & 3.9121 & 3.8132 & -2.6 \\
\hline & 3 & 6.6113 & 7.8548 & 15.8 & 6.5092 & 7.7613 & 16.1 & 6.479 & 7.7536 & 16.4 & 5.9321 & 6.8610 & 13.5 \\
\hline & 4 & 8.5186 & 10.9955 & 22.5 & 8.1882 & 10.296 & 20.5 & 7.9608 & 9.5163 & 16.3 & 7.1856 & 8.6368 & 16.8 \\
\hline & 5 & 10.1584 & 14.1372 & 28.1 & 9.9341 & 13.2402 & 24.9 & 9.8889 & 13.014 & 24.0 & 9.2159 & 12.0138 & 23.3 \\
\hline \multirow{5}{*}{10} & 1 & 1.8677 & 1.8751 & 0.4 & 1.6557 & 1.6603 & 0.3 & 1.6457 & 1.6308 & -0.9 & 1.6452 & 1.6303 & -0.9 \\
\hline & 2 & 4.5724 & 4.6941 & 2.6 & 4.5450 & 4.6590 & 2.4 & 4.3632 & 4.178 & -4.4 & 4.3153 & 4.1448 & -4.1 \\
\hline & 3 & 7.4154 & 7.8548 & 5.6 & 7.3492 & 7.7913 & 5.6 & 7.3447 & 7.7881 & 5.7 & 6.9612 & 7.3250 & 4.9 \\
\hline & 4 & 9.9873 & 10.9955 & 9.1 & 9.6142 & 10.4963 & 8.4 & 9.3903 & 9.8721 & 4.8 & 8.7555 & 9.1851 & 4.6 \\
\hline & 5 & 12.3224 & 14.1372 & 12.8 & 11.9264 & 3.4203 & 11.1 & 11.8842 & 13.3107 & 10.7 & 11.2005 & 12.4725 & 0.2 \\
\hline \multirow{5}{*}{20} & 1 & 732 & 8751 & 0.1 & 1.7502 & 1.7516 & 0.1 & 1.7435 & 1.7320 & -0.7 & 1.7432 & 1.7316 & -0.7 \\
\hline & 2 & & & 0. & & & 0.6 & & & -3.2 & 4.5139 & & -3.1 \\
\hline & 3 & & & 1.6 & & & 1.6 & & 7.8151 & 1.6 & 7.4669 & 7.5792 & 1.5 \\
\hline & 4 & 10.6862 & 10.9955 & 2.8 & .3983 & 10.6816 & 2.6 & 10.2317 & 10.2403 & 0.1 & 9.7774 & 9.7825 & 0.1 \\
\hline & 5 & 13.5319 & 14.1372 & 4.3 & 13.1149 & 13.6321 & 3.8 & 3.0995 & 13.5955 & 3.6 & 12.5261 & 12.9795 & 3.5 \\
\hline
\end{tabular}




\section{CONCLUSION} follow:

Summarizing results obtained in the present study the conclusions can be made as

A closed form solution has been conducted for free vibration of Timoshenko beam with arbitrary number of cracks. This solution is straightforward to derive an explicit expression for frequency equation and mode shapes of multiple cracked Timoshenko beams;

Analysis of natural frequencies obtained from the frequency equation shows that the Timoshenko beam theory is useful for vibration analysis of not only short or thick cracked beams but also the long or slender ones, while the Euler-Bernoulli beam theory is applicable only for long and slender beams;

Nevertheless, sensitivities of natural frequencies to cracks computed by the EBT and TBT are the same for beams of slenderness ratio $(L / h)$ greater than 20 and for the ratio less than 20 natural frequencies computed by the EBT are more sensitive to cracks than those computed by TBT;

The obtained closed-form solution can be used for vibration analysis and crack identification of more complicated structures such as stepped multispan beams or framed structures with cracks that is a subject of next studies of the authors.

\section{REFERENCES}

[1] I. Elishakoff, J. Kaplunov, and E. Nolde. Celebrating the centenary of Timoshenko's study of effects of shear deformation and rotary inertia. Applied Mechanics Reviews, 67, (6), (2015). doi:10.1115/1.4031965.

[2] L. Majkut. Free and forced vibrations of Timoshenko beams described by single difference equation. Journal of Theoretical and Applied Mechanics, 47, (1), (2009), pp. 193-210, http://ptmts.org.pl/jtam/index.php/jtam/article/view/v47n1p193.

[3] I. Karnovsky and O. Lebed. Formulas for structural dynamics: tables, graphs and solutions. McGraw Hill, Inc., (2000).

[4] T. Kocatürk and M. Şimşek. Free vibration analysis of Timoshenko beams under various boundary conditions. Journal of Engineering and Natural Sciences, 1, (2005), pp. 30-40, http://eds.yildiz.edu.tr/ArticleContent/Journal/sigma/Volumes/2005/Issues/Regular1/YTUJENS-2005-23-1.311.pdf.

[5] R. D. Adams, P. Cawley, C. J. Pye, and B. J. Stone. A vibration technique for non-destructively assessing the integrity of structures. Journal of Mechanical Engineering Science, 20, (2), (1978), pp. 93-100. doi:10.1243/jmes_jour_1978_020_016_02.

[6] R. Y. Liang, J. Hu, and F. Choy. Quantitative NDE technique for assessing damages in beam structures. Journal of Engineering Mechanics, 118, (7), (1992), pp. 1468-1487. doi:10.1061/(asce)0733-9399(1992)118:7(1468).

[7] Y. Narkis. Identification of crack location in vibrating simply supported beams. Journal of Sound and Vibration, 172, (4), (1994), pp. 549-558. doi:10.1006/jsvi.1994.1195.

[8] T. G. Chondros, A. D. Dimarogonas, and J. Yao. A continuous cracked beam vibration theory. Journal of Sound and Vibration, 215, (1), (1998), pp. 17-34. doi:10.1006/jsvi.1998.1640.

[9] N. T. Khiem and T. V. Lien. A simplified method for natural frequency analysis of a multiple cracked beam. Journal of Sound and Vibration, 245, (4), (2001), pp. 737-751. doi:10.1006/jsvi.2001.3585. 
[10] N. T. Khiem and H. T. Tran. A procedure for multiple crack identification in beam-like structures from natural vibration mode. Journal of Vibration and Control, 20, (9), (2014), pp. 14171427. doi:10.1177/1077546312470478.

[11] S. Caddemi and I. Calio. Exact closed-form solution for the vibration modes of the EulerBernoulli beam with multiple open cracks. Journal of Sound and Vibration, 327, (3), (2009), pp. 473-489. doi:10.1016/j.jsv.2009.07.008.

[12] N. T. Khiem and T. T. Hai. A closed-form solution for free vibration of beams with arbitrary number of cracks. In Proceedings of the Scientific Conference dedicated to 35th Anniversary of Vietnam Academy of Science and Technology, Vol. 1, Hanoi, Vietnam, (2010). pp. 30-42.

[13] T. C. Tsai and Y. Z. Wang. Vibration analysis and diagnosis of a cracked shaft. Journal of Sound and Vibration, 192, (3), (1996), pp. 607-620. doi:10.1006/jsvi.1996.0209.

[14] S. P. Lele and S. K. Maiti. Modelling of transverse vibration of short beams for crack detection and measurement of crack extension. Journal of Sound and Vibration, 257, (3), (2002), pp. 559583. doi:10.1006/jsvi.2002.5059.

[15] Q. S. Li. Vibratory characteristics of Timoshenko beams with arbitrary number of cracks. Journal of Engineering Mechanics, 129, (11), (2003), pp. 1355-1359. doi:10.1061/(asce)07339399(2003)129:11(1355).

[16] J. A. Loya, L. Rubio, and J. Fernández-Sáez. Natural frequencies for bending vibrations of Timoshenko cracked beams. Journal of Sound and Vibration, 290, (3), (2006), pp. 640-653. doi:10.1016/j.jsv.2005.04.005.

[17] A. S. J. Swamidas, X. Yang, and R. Seshadri. Identification of cracking in beam structures using Timoshenko and Euler formulations. Journal of Engineering Mechanics, 130, (11), (2004), pp. 1297-1308. doi:10.1061/(asce)0733-9399(2004)130:11(1297).

[18] K. Aydin. Influence of crack and slenderness ratio on the eigenfrequencies of Euler-Bernoulli and Timoshenko beams. Mechanics of Advanced Materials and Structures, 20, (5), (2013), pp. 339-352. doi:10.1080/15376494.2011.627635.

[19] N. Khaji, M. Shafiei, and M. Jalalpour. Closed-form solutions for crack detection problem of Timoshenko beams with various boundary conditions. International Journal of Mechanical Sciences, 51, (9), (2009), pp. 667-681. doi:10.1016/j.ijmecsci.2009.07.004.

[20] S. Fekrazadeh and N. Khaji. An analytical method for crack detection of Timoshenko beams with multiple open cracks using a test mass. European Journal of Environmental and Civil Engineering, 21, (1), (2017), pp. 24-41. doi:10.1080/19648189.2015.1090929. 